\title{
Multiplication and Translation Operators on the Fock Spaces for the $q$-Modified Bessel Function
}

\author{
Fethi Soltani \\ Higher College of Technology and Informatics, Street of the Employers, Tunis, Tunisia \\ E-mail:fethisoltani10@yahoo.com \\ Received February 28, 2011; revised April 8, 2011; accepted April 20, 2011
}

\section{Abstract}

We study the multiplication operator $M$ by $z^{2}$ and the $q$-Bessel operator $\Delta_{q, \alpha}$ on a Hilbert spaces $\mathbb{F}_{q, \alpha}$ of entire functions on the disk $D\left(o, \frac{1}{1-q}\right), 0<q<1$; and we prove that these operators are adjoint-operators and continuous from $\mathbb{F}_{q, \alpha}$ into itself. Next, we study a generalized translation operators on $\mathbb{F}_{q, \alpha}$.

Keywords: Generalized $q$-Fock Spaces, $q-I_{\alpha}$ Modified Bessel Function, $q$-Bessel Operator, Multiplication Operator, $q$-Translation Operators

\section{Introduction}

In 1961, Bargmann [1] introduced a Hilbert space $\mathbb{F}$ of entire functions $f(z)=\sum_{n=0}^{\infty} a_{n} z^{n}$ on $\mathbb{C}$ such that

$$
\|f\|_{\mathbb{F}}^{2}:=\sum_{n=0}^{\infty}\left|a_{n}\right|^{2} n !<\infty
$$

On this space the author studied the differential operator $D=\mathrm{d} / \mathrm{d} z$ and the multiplication operator by $z$, and proved that these operators are densely defined, closed and adjoint-operators on $\mathbb{F}$ (see [1]).

Next, the Hilbert space $\mathbb{F}$ is called Segal-Bargmann space or Fock space and it was the aim of many works [2].

In 1984, Cholewinski [3] introduced a Hilbert space $\mathbb{F}_{\alpha}$ of even entire functions on $\mathbb{C}$, where the inner product is weighted by the modified Macdonald function. On $\mathbb{F}_{\alpha}$ the Bessel operator

$$
\Delta_{\alpha}:=\frac{\mathrm{d}^{2}}{\mathrm{~d} z^{2}}+\frac{2 \alpha+1}{z} \frac{\mathrm{d}}{\mathrm{d} z}, \quad \alpha>-1 / 2
$$

and the multiplication by $z^{2}$ are densely defined, closed and adjoint-operators.

"Author partially supported by DGRST project 04/UR/15-02 and CMCU program 10G 1503.
In this paper, we consider the $q-I_{\alpha}$ modified Bessel function:

$$
I_{\alpha}\left(x ; q^{2}\right):=\sum_{n=0}^{\infty} \frac{x^{2 n}}{b_{2 n}\left(\alpha ; q^{2}\right)}
$$

where $b_{2 n}\left(\alpha ; q^{2}\right)$ are given later in Section 2. We define the $q$-Fock space $\mathbb{F}_{q, \alpha}$ as the Hilbert space of even entire functions $f(z)=\sum_{n=0}^{\infty} a_{n} z^{2 n}$ on the disk $D\left(o, \frac{1}{1-q}\right)$ of center $o$ and radius $\frac{1}{1-q}$, and such that

$$
\|f\|_{\mathbb{F}_{q, \alpha}}^{2}:=\sum_{n=0}^{\infty}\left|a_{n}\right|^{2} b_{2 n}\left(\alpha ; q^{2}\right)<\infty
$$

Let $f$ and $g$ be in $\mathbb{F}_{q, \alpha}$, such that $f(z)=\sum_{n=0}^{\infty} a_{n} z^{2 n}$ and $g(z)=\sum_{n=0}^{\infty} c_{n} z^{2 n}$, the inner product is given by

$$
\langle f, g\rangle_{\mathbb{F}_{q, \alpha}}=\sum_{n=0}^{\infty} a_{n} \overline{c_{n}} b_{2 n}\left(\alpha ; q^{2}\right)<\infty
$$

Next, we consider the multiplication operator $M$ by $z^{2}$ and the $q$-Bessel operator $\Delta_{q, \alpha}$ on the Fock space $\mathbb{F}_{q, \alpha}$, and we prove that these operators are continuous from $\mathbb{F}_{q, \alpha}$ into itself, and satisfy: 


$$
\begin{gathered}
\left\|\Delta_{q, \alpha} f\right\|_{\mathbb{F}_{q, \alpha}} \leq \frac{1}{1-q}\|f\|_{\mathbb{F}_{q, \alpha}} \\
\|M f\|_{\mathbb{F}_{q, \alpha}} \leq \frac{1}{1-q}\|f\|_{\mathbb{F}_{q, \alpha}}
\end{gathered}
$$

Then, we prove that these operators are adjointoperators on $\mathbb{F}_{q, \alpha}$ :

$$
\langle M f, g\rangle_{\mathbb{F}_{q, \alpha}}=\left\langle f, \Delta_{q, \alpha} g\right\rangle_{\mathbb{F}_{q, \alpha}} ; f, g \in \mathbb{F}_{q, \alpha}
$$

Lastly, we define and study on the Fock space $\mathbb{F}_{q, \alpha}$, the $q$-translation operators:

$$
T_{z} f(w):=I_{\alpha}\left(z \Delta_{q, \alpha}^{1 / 2} ; q^{2}\right) f(w) ; \quad w, z \in D\left(o, \frac{1}{1-q}\right)
$$

and the generalized multiplication operators:

$$
M_{z} f(w):=I_{\alpha}\left(z M^{1 / 2} ; q^{2}\right) f(w) ; \quad w, z \in D\left(o, \frac{1}{1-q}\right) .
$$

Using the previous results, we deduce that the operators $T_{z}$ and $M_{z}$, for $z \in D\left(o, \frac{1}{1-q}\right)$, are continuous from $\mathbb{F}_{q, \alpha}$ into itself, and satisfy:

$$
\begin{gathered}
\left\|T_{z} f\right\|_{\mathbb{F}_{q, \alpha}} \leq I_{\alpha}\left(\frac{|z|}{\sqrt{1-q}} ; q^{2}\right)\|f\|_{\mathbb{F}_{q, \alpha}} \\
\left\|M_{z} f\right\|_{\mathbb{F}_{q, \alpha}} \leq I_{\alpha}\left(\frac{|z|}{\sqrt{1-q}} ; q^{2}\right)\|f\|_{\mathbb{F}_{q, \alpha}}
\end{gathered}
$$

\section{The $q$-Fock Spaces $\mathbb{F}_{q, \alpha}$}

Let $a$ and $q$ be real numbers such that $0<q<1$; the $q$-shifted factorial are defined by

$$
(a ; q)_{0}:=1, \quad(a ; q)_{n}:=\prod_{i=0}^{n-1}\left(1-a q^{i}\right), n=1,2, \cdots, \infty
$$

Jackson [5] defined the $q$-analogue of the Gamma function as

$$
\Gamma_{q}(x):=\frac{(q ; q)_{\infty}}{\left(q^{x} ; q\right)_{\infty}}(1-q)^{1-x}, \quad x \neq 0,-1,-2, \cdots
$$

It satisfies the functional equation

$$
\Gamma_{q}(x+1)=\frac{1-q^{x}}{1-q} \Gamma_{q}(x), \quad \Gamma_{q}(1)=1
$$

and tends to $\Gamma(x)$ when $q$ tends to $1^{-}$. In particular, for $n=1,2, \cdots$, we have

$$
\Gamma_{q}(n+1)=\frac{(q ; q)_{n}}{(1-q)^{n}}
$$

The $q$-combinatorial coefficients are defined for $n, k \in \mathbb{N}, k=0, \cdots, n$, by

$$
\left(\begin{array}{l}
n \\
k
\end{array}\right)_{q}:=\frac{(q ; q)_{n}}{(q ; q)_{k}(q ; q)_{n-k}}=\frac{\Gamma_{q}(n+1)}{\Gamma_{q}(k+1) \Gamma_{q}(n-k+1)}
$$

The $q$-derivative $D_{q} f$ of a suitable function $f$ (see [6]) is given by

$$
D_{q} f(x):=\frac{f(x)-f(q x)}{(1-q) x}, \quad x \neq 0
$$

and $D_{q} f(0)=f^{\prime}(0)$ provided $f^{\prime}(0)$ exists.

If $f$ is differentiable then $D_{q} f(x)$ tends to $f^{\prime}(x)$ as $q \rightarrow 1^{-}$.

Taking account of the paper [4] and the same way, we define the $q-I_{\alpha}$ modified Bessel function by

$$
I_{\alpha}\left(x ; q^{2}\right):=\sum_{n=0}^{\infty} \frac{x^{2 n}}{b_{2 n}\left(\alpha ; q^{2}\right)}
$$

where

$$
b_{2 n}\left(\alpha ; q^{2}\right):=\frac{(1+q)^{2 n} \Gamma_{q^{2}}(n+1) \Gamma_{q^{2}}(n+\alpha+1)}{\Gamma_{q^{2}}(\alpha+1)}
$$

$$
\begin{aligned}
& \text { If we put } U_{n}=\frac{1}{b_{2 n}\left(\alpha ; q^{2}\right)} \text {, then } \\
& \qquad \frac{U_{n}}{U_{n+1}} \rightarrow \frac{1}{(1-q)^{2}}, \quad q \rightarrow 1^{-}
\end{aligned}
$$

Thus, the $q-I_{\alpha}$ modified Bessel function is defined on $D\left(o, \frac{1}{(1-q)^{2}}\right)$ and tends to the $I_{\alpha}$ modified Bessel function as $q \rightarrow 1^{-}$.

In [4], the authors study in great detail the $q$-Bessel operator denoted by

$$
\Delta_{q, \alpha} f(x):=D_{q}^{2} f(x)+\frac{[2 \alpha+1]_{q}}{x} D_{q} f(q x)
$$

where

$$
[2 \alpha+1]_{q}:=\frac{1-q^{2 \alpha+1}}{1-q}
$$

The $q$-Bessel operator tends to the Bessel operator $\Delta_{\alpha}$ as $q \rightarrow 1^{-}$.

Lemma 1: 1) The function $I_{\alpha}\left(\lambda \cdot ; q^{2}\right), \lambda \in D\left(o, \frac{1}{1-q}\right)$, is the unique analytic solution of the q-problem:

$$
\Delta_{q, \alpha} y(x)=\lambda^{2} y(x), y(0)=1 \text { and } D_{q} y(0)=0
$$


2) For $n \in \mathbb{N}$, we have

$$
\Delta_{q, \alpha} z^{2 n}=\frac{b_{2 n}\left(\alpha ; q^{2}\right)}{b_{2(n-1)}\left(\alpha ; q^{2}\right)} z^{2(n-1)}, \quad n \geq 1
$$

3) The constants $b_{2 n}\left(\alpha ; q^{2}\right), \quad n \in \mathbb{N}$ satisfy the following relation:

$$
b_{2 n+2}\left(\alpha ; q^{2}\right)=[2 n+2]_{q}[2 n+2 \alpha+2]_{q} b_{2 n}\left(\alpha ; q^{2}\right)
$$

Let $\alpha \geq-1 / 2$. The $q$-Fock space $\mathbb{F}_{q, \alpha}$ is the Hilbert space of even entire functions $f(z)=\sum_{n=0}^{\infty} a_{n} z^{2 n}$ on $D\left(o, \frac{1}{1-q}\right)$, such that

$$
\|f\|_{\mathbb{F}_{q, \alpha}}^{2}:=\sum_{n=0}^{\infty}\left|a_{n}\right|^{2} b_{2 n}\left(\alpha ; q^{2}\right)<\infty
$$

where $b_{2 n}\left(\alpha ; q^{2}\right)$ is given by (2).

The inner product in $\mathbb{F}_{q, \alpha}$ is given for

$$
\begin{array}{r}
f(z)=\sum_{n=0}^{\infty} a_{n} z^{2 n} \text { and } g(z)=\sum_{n=0}^{\infty} c_{n} z^{2 n} \text { by } \\
\langle f, g\rangle_{\mathbb{F}_{q, \alpha}}=\sum_{n=0}^{\infty} a_{n} \overline{c_{n}} b_{2 n}\left(\alpha ; q^{2}\right)
\end{array}
$$

Remark 1: If $q \rightarrow 1^{-}$, the space $\mathbb{F}_{q, \alpha}$ agrees with the generalized Fock space associated to the Bessel operator (see [3]).

Theorem 1: The function $\kappa_{q, \alpha}$ given for

$$
\begin{aligned}
w, z & \in D\left(o, \frac{1}{1-q}\right), \text { by } \\
\kappa_{q, \alpha}(w, z) & =I_{\alpha}\left(\bar{w} z ; q^{2}\right)
\end{aligned}
$$

is a reproducing kernel for the $q$-Fock space $\mathbb{F}_{q, \alpha}$, that is:

1) For all $w \in D\left(o, \frac{1}{1-q}\right)$, the function $z \rightarrow \kappa_{q, \alpha}(w, z)$ belongs to $\mathbb{F}_{q, \alpha}$.

2) For all $w \in D\left(o, \frac{1}{1-q}\right)$ and $f \in \mathbb{F}_{q, \alpha}$, we have

$$
\left\langle f, \kappa_{q, \alpha}(w, .)\right\rangle_{\mathbb{F}_{q, \alpha}}=f(w)
$$

Remark 2: From Theorem 1, 2), for $f \in \mathbb{F}_{q, \alpha}$ and $w \in D\left(o, \frac{1}{1-q}\right)$, we have

$$
|f(w)| \leq\left\|\kappa_{q, \alpha}(w, .)\right\|_{\mathbb{F}_{q, \alpha}}\|f\|_{\mathbb{F}_{q, \alpha}}=\left[I_{\alpha}\left(|w|^{2} ; q^{2}\right)\right]^{1 / 2}\|f\|_{\mathbb{F}_{q, \alpha}}
$$

\section{Multiplication and $q$-Bessel Operators on} $\mathbb{F}_{q, \alpha}$

On $\mathbb{F}_{q, \alpha}$, we consider the multiplication operators $M$ and $N_{q}$ given by

$$
\begin{gathered}
M f(z):=z^{2} f(z) \\
N_{q} f(z):=z D_{q} f(z)=\frac{f(z)-f(q z)}{1-q}
\end{gathered}
$$

We denote also by $\Delta_{q, \alpha}$ the $q$-Bessel operator defined for entire functions on $D\left(o, \frac{1}{1-q}\right)$.

We write

$$
\left[\Delta_{q, \alpha}, M\right]=\Delta_{q, \alpha} M-M \Delta_{q, \alpha}
$$

By straightforward calculation we obtain the following result.

Lemma 2: $\left[\Delta_{q, \alpha}, M\right]=(1+q)[2 \alpha+2]_{q} B_{q^{2}}+W_{q, \alpha}$, where

$$
B_{q}(z):=f(q z)
$$

and

$$
W_{q, \alpha} f(z):=(1+q)\left(1+q^{2 \alpha}\right) q z D_{q}(f)(q z)
$$

Remark 3: The Lemma 2 is the analogous commutation rule of Cholewinski [3]. When $q \rightarrow 1^{-}$, then $\left[\Delta_{q, \alpha}, M\right]$ tends to $4(\alpha+1) I+4 z \frac{\mathrm{d}}{\mathrm{d} z}$, where $I$ is the identity operator.

Lemma 3: If $f \in \mathbb{F}_{q, \alpha}$ then $B_{q} f, N_{q} f$ and $W_{q, \alpha} f$ belong to $\mathbb{F}_{q, \alpha}$, and

1) $\left\|B_{q} f\right\|_{\mathbb{F}_{q, \alpha}} \leq\|f\|_{\mathbb{F}_{q, \alpha}}$,

2) $\left\|N_{q} f\right\|_{\mathbb{F}_{q, \alpha}} \leq \frac{1}{1-q}\|f\|_{\mathbb{F}_{q, \alpha}}$,

3) $\left\|W_{q, \alpha} f\right\|_{\mathbb{F}_{q, \alpha}} \leq \frac{(1+q)\left(1+q^{2 \alpha}\right)}{1-q}\|f\|_{\mathbb{F}_{q, \alpha}}$.

Proof. Let $f(z)=\sum_{n=0}^{\infty} a_{n} z^{2 n} \in \mathbb{F}_{q, \alpha}$, then

$$
\begin{aligned}
& B_{q} f(z)=f(q z)=\sum_{n=0}^{\infty} a_{n} q^{2 n} z^{2 n} \\
& N_{q} f(z)=\frac{f(z)-f(q z)}{1-q}=\sum_{n=0}^{\infty} a_{n}[2 n]_{q} z^{n}
\end{aligned}
$$

and from (4), we obtain 


$$
\begin{aligned}
\left\|B_{q} f\right\|_{\mathbb{F}_{q, \alpha}}^{2} & =\sum_{n=0}^{\infty}\left|a_{n}\right|^{2} q^{4 n} b_{2 n}\left(\alpha ; q^{2}\right) \\
& \leq \sum_{n=0}^{\infty}\left|a_{n}\right|^{2} b_{2 n}\left(\alpha ; q^{2}\right)=\|f\|_{\mathbb{F}_{q, \alpha}}^{2}
\end{aligned}
$$

and

$$
\left\|N_{q} f\right\|_{\mathbb{F}_{q, \alpha}}^{2}=\sum_{n=0}^{\infty}\left|a_{n}\right|^{2}\left([2 n]_{q}\right)^{2} b_{2 n}\left(\alpha ; q^{2}\right)
$$

Using the fact that $[2 n]_{q} \leq \frac{1}{1-q}$, we deduce

$$
\left\|N_{q} f\right\|_{\mathbb{F}_{q, \alpha}}^{2} \leq \frac{1}{(1-q)^{2}} \sum_{n=0}^{\infty}\left|a_{n}\right|^{2} b_{2 n}\left(\alpha ; q^{2}\right)=\frac{1}{(1-q)^{2}}\|f\|_{\mathbb{F}_{q, \alpha}}^{2}
$$

On the other hand from (6), we have

$$
W_{q, \alpha} f(z)=(1+q)\left(1+q^{2 \alpha}\right) \sum_{n=1}^{\infty} a_{n}[2 n]_{q} q^{2 n} z^{2 n}
$$

and

$$
\begin{aligned}
\left\|W_{q, \alpha} f\right\|_{\mathbb{F}_{q, \alpha}}^{2} & =\left[(1+q)\left(1+q^{2 \alpha}\right)\right]^{2} \\
& \cdot \sum_{n=1}^{\infty}\left|a_{n}\right|^{2}\left([2 n]_{q}\right)^{2} q^{4 n} b_{2 n}\left(\alpha ; q^{2}\right)
\end{aligned}
$$

Using the fact that $[2 n]_{q} \leq \frac{1}{1-q}$, we deduce that

$$
\left\|W_{q, \alpha} f\right\|_{\mathbb{F}_{q, \alpha}}^{2} \leq \frac{\left[(1+q)\left(1+q^{2 \alpha}\right)\right]^{2}}{(1-q)^{2}} \sum_{n=1}^{\infty}\left|a_{n}\right|^{2} b_{2 n}\left(\alpha ; q^{2}\right)
$$

Therefore, we conclude that

$$
\left\|W_{q, \alpha} f\right\|_{\mathbb{F}_{q, \alpha}} \leq \frac{(1+q)\left(1+q^{2 \alpha}\right)}{1-q}\|f\|_{\mathbb{F}_{q, \alpha}}
$$

which completes the proof of the Lemma.

Theorem 2: If $f \in \mathbb{F}_{q, \alpha}$ then $\Delta_{q, \alpha} f$ and $M f$ belong to $\mathbb{F}_{q, \alpha}$, and we have

1) $\left\|\Delta_{q, \alpha} f\right\|_{\mathbb{F}_{q, \alpha}} \leq \frac{1}{1-q}\|f\|_{\mathbb{F}_{q, \alpha}}$,

2) $\|M f\|_{\mathbb{F}_{q, \alpha}} \leq \frac{1}{1-q}\|f\|_{\mathbb{F}_{q, \alpha}}$.

Proof. Let $f(z)=\sum_{n=0}^{\infty} a_{n} z^{2 n} \in \mathbb{F}_{q, \alpha}$.

1) From Lemma 1, 2),

$$
\begin{aligned}
\Delta_{q, \alpha} f(z) & =\sum_{n=1}^{\infty} a_{n} \frac{b_{2 n}\left(\alpha ; q^{2}\right)}{b_{2(n-1)}\left(\alpha ; q^{2}\right)} z^{2(n-1)} \\
& =\sum_{n=0}^{\infty} a_{n+1} \frac{b_{2 n+2}\left(\alpha ; q^{2}\right)}{b_{2 n}\left(\alpha ; q^{2}\right)} z^{2 n}
\end{aligned}
$$

Then from (10), we get

$$
\left\|\Delta_{q, \alpha} f\right\|_{\mathbb{F}_{q, \alpha}}^{2}=\sum_{n=0}^{\infty}\left|a_{n+1}\right|^{2} \frac{b_{2 n+2}\left(\alpha ; q^{2}\right)}{b_{2 n}\left(\alpha ; q^{2}\right)} b_{2 n+2}\left(\alpha ; q^{2}\right)
$$

Using Lemma 1, 3), we obtain

$\left\|\Delta_{q, \alpha} f\right\|_{\mathbb{F}_{q, \alpha}}^{2}=\sum_{n=0}^{\infty}\left|a_{n+1}\right|^{2}[2 n+2]_{q}[2 n+2 \alpha+2]_{q} b_{2 n+2}\left(\alpha ; q^{2}\right)$

and consequently,

$$
\left\|\Delta_{q, \alpha} f\right\|_{\mathbb{F}_{q, \alpha}}^{2}=\sum_{n=1}^{\infty}\left|a_{n}\right|^{2}[2 n]_{q}[2 n+2 \alpha]_{q} b_{2 n}\left(\alpha ; q^{2}\right)
$$

Using the fact that $[2 n]_{q}[2 n+2 \alpha]_{q} \leq \frac{1}{(1-q)^{2}}$, we obtain

$$
\left\|\Delta_{q, \alpha} f\right\|_{\mathbb{F}_{q, \alpha}} \leq \frac{1}{1-q}\left[\sum_{n=1}^{\infty}\left|a_{n}\right|^{2} b_{2 n}\left(\alpha ; q^{2}\right)\right]^{1 / 2}=\frac{1}{1-q}\|f\|_{\mathbb{F}_{q, \alpha}}
$$

2) On the other hand, since

$$
M f(z)=\sum_{n=1}^{\infty} a_{n-1} z^{2 n}
$$

then

$$
\|M f\|_{\mathbb{F}_{q, \alpha}}^{2}=\sum_{n=1}^{\infty}\left|a_{n-1}\right|^{2} b_{2 n}\left(\alpha ; q^{2}\right)=\sum_{n=0}^{\infty}\left|a_{n}\right|^{2} b_{2 n+2}\left(\alpha ; q^{2}\right)
$$

By Lemma 1, 3), we deduce

$$
\|M f\|_{\mathbb{F}_{q, \alpha}}^{2}=\sum_{n=0}^{\infty}\left|a_{n}\right|^{2}[2 n+2]_{q}[2 n+2 \alpha+2]_{q} b_{2 n}\left(\alpha ; q^{2}\right)
$$

Using the fact that $[2 n+2]_{q}[2 n+2 \alpha+2]_{q} \leq \frac{1}{(1-q)^{2}}$, we obtain

$$
\|M f\|_{\mathbb{F}_{q, \alpha}} \leq \frac{1}{1-q}\|f\|_{\mathbb{F}_{q, \alpha}}
$$

We deduce also the following norm equalities.

Theorem 3: If $f \in \mathbb{F}_{q, \alpha}$ then

1) $\left\langle f, W_{q, \alpha} f\right\rangle_{\mathbb{F}_{q, \alpha}}=(1+q)\left(1+q^{2 \alpha}\right)\left\langle N_{q} f, B_{q} f\right\rangle_{\mathbb{F}_{q, \alpha}}$,

2) $\left\|\Delta_{q, \alpha} f\right\|_{\mathbb{F}_{q, \alpha}}^{2}=\left\|N_{q} f\right\|_{\mathbb{F}_{q, \alpha}}^{2}+[2 \alpha]_{q}\left\langle N_{q} f, B_{q} f\right\rangle_{\mathbb{F}_{q, \alpha}}$,

3) $\|M f\|_{\mathbb{F}_{q, \alpha}}^{2}=\left\|N_{q} f\right\|_{\mathbb{F}_{q, \alpha}}^{2}+(1+q)[2 \alpha+2]_{q}\left\|B_{q} f\right\|_{\mathbb{F}_{q, \alpha}}^{2}$

$$
+\left(1+q+[2 \alpha+2]_{q}\right)\left\langle N_{q} f, B_{q} f\right\rangle_{\mathbb{F}_{q, \alpha}},
$$

4) $\|M f\|_{\mathbb{F}_{q, \alpha}}^{2}=\left\|\Delta_{q, \alpha} f\right\|_{\mathbb{F}_{q, \alpha}}^{2}+(1+q)[2 \alpha+2]_{q}\left\|B_{q} f\right\|_{\mathbb{F}_{q, \alpha}}^{2}$

$$
+\left\langle f, W_{q, \alpha} f\right\rangle_{\mathbb{F}_{q, \alpha}} \text {. }
$$


Proof. Let $f(z)=\sum_{n=0}^{\infty} a_{n} z^{2 n} \in \mathbb{F}_{q, \alpha}$.

1) Follows from (7), (8) and (9).

2) From (11), we get

$$
\left\|\Delta_{q, \alpha} f\right\|_{\mathbb{F}_{q, \alpha}}^{2}=\sum_{n=0}^{\infty}\left|a_{n}\right|^{2}[2 n]_{q}[2 n+2 \alpha]_{q} b_{2 n}\left(\alpha ; q^{2}\right)
$$

Using the fact $[2 n+2 \alpha]_{q}=[2 n]_{q}+q^{2 n}[2 \alpha]_{q}$, we deduce

$$
\left\|\Delta_{q, \alpha} f\right\|_{\mathbb{F}_{q, \alpha}}^{2}=\left\|N_{q} f\right\|_{\mathbb{F}_{q, \alpha}}^{2}+[2 \alpha]_{q}\left\langle N_{q} f, B_{q} f\right\rangle_{\mathbb{F}_{q, \alpha}}
$$

3) By (13) and using the fact that

$$
\begin{aligned}
& {[2 n+2]_{q}[2 n+2 \alpha+2]_{q}} \\
& =\left([2 n]_{q}\right)^{2}+\left(1+q+[2 \alpha+2]_{q}\right) q^{2 n}[2 n]_{q} \\
& \quad+(1+q)[2 \alpha+2]_{q} q^{4 n}
\end{aligned}
$$

we obtain

$$
\begin{aligned}
\|M f\|_{\mathbb{F}_{q, \alpha}}^{2}= & \left\|N_{q} f\right\|_{\mathbb{F}_{q, \alpha}}^{2}+(1+q)[2 \alpha+2]_{q}\left\|B_{q} f\right\|_{\mathbb{F}_{q, \alpha}}^{2} \\
& +\left(1+q+[2 \alpha+2]_{q}\right)\left\langle N_{q} f, B_{q} f\right\rangle_{\mathbb{F}_{q, \alpha}}
\end{aligned}
$$

4) Follows directly from 1), 2) and 3).

Remark 4: Let $f(z)=\sum_{n=0}^{\infty} a_{n} z^{2 n} \in \mathbb{F}_{q, \alpha}$. Since $\left\langle f, W_{q, \alpha} f\right\rangle_{\mathbb{F}_{q, \alpha}} \geq 0$, then

$$
\|M f\|_{\mathbb{F}_{q, \alpha}}^{2} \geq(1+q)[2 \alpha+2]_{q}\left\|B_{q} f\right\|_{\mathbb{F}_{q, \alpha}}^{2}
$$

Therefore $M f=0$ implies that $f=0$. Then $M: \mathbb{F}_{q, \alpha} \rightarrow \mathbb{F}_{q, \alpha}$ is an injective continuous operator on $\mathbb{F}_{q, \alpha}$.

Proposition 1: The operators $M$ and $\Delta_{q, \alpha}$ are adjoint-operators on $\mathbb{F}_{q, \alpha}$; and for all $f, g \in \mathbb{F}_{q, \alpha}$, we have

$$
\langle M f, g\rangle_{\mathbb{F}_{q, \alpha}}=\left\langle f, \Delta_{q, \alpha} g\right\rangle_{\mathbb{F}_{q, \alpha}}
$$

Proof. Consider $f(z)=\sum_{n=0}^{\infty} a_{n} z^{2 n}$ and $g(z)=\sum_{n=0}^{\infty} c_{n} z^{2 n}$ in $\mathbb{F}_{q, \alpha}$. From (10) and (12),

$$
\Delta_{q, \alpha} g(z)=\sum_{n=0}^{\infty} c_{n+1} \frac{b_{2 n+2}\left(\alpha ; q^{2}\right)}{b_{2 n}\left(\alpha ; q^{2}\right)} z^{2 n}
$$

and

$$
M f(z)=\sum_{n=1}^{\infty} a_{n-1} z^{2 n}
$$

Thus from (5), we get

$$
\begin{aligned}
\langle M f, g\rangle_{\mathbb{F}_{q, \alpha}} & =\sum_{n=1}^{\infty} a_{n-1} \overline{c_{n}} b_{2 n}\left(\alpha ; q^{2}\right) \\
& =\sum_{n=0}^{\infty} a_{n} \overline{c_{n+1}} b_{2 n+2}\left(\alpha ; q^{2}\right) \\
& =\left\langle f, \Delta_{q, \alpha} g\right\rangle_{\mathbb{F}_{q, \alpha}}
\end{aligned}
$$

which gives the result.

\section{Generalized Multiplication and Translation Operators on $\mathbb{F}_{q, \alpha}$}

In this section, we study a generalized multiplication and translation operators on $\mathbb{F}_{q, \alpha}$.

Definition 2: For $f \in \mathbb{F}_{q, \alpha}$, and $w, z \in D\left(o, \frac{1}{1-q}\right)$, we define:

-The $q$-translation operators on $\mathbb{F}_{q, \alpha}$, by

$$
\tau_{z} f(w):=\sum_{n=0}^{\infty} \frac{\Delta_{q, \alpha}^{n} f(w)}{b_{2 n}\left(\alpha ; q^{2}\right)} z^{2 n}
$$

-The generalized multiplication operators on $\mathbb{F}_{q, \alpha}$, by

$$
M_{z} f(w):=\sum_{n=0}^{\infty} \frac{M^{n} f(w)}{b_{2 n}\left(\alpha ; q^{2}\right)} z^{2 n}
$$

For $w, z \in D\left(o, \frac{1}{1-q}\right)$, the function $I\left(. ; q^{2}\right)$ satisfies the following product formulas:

$$
\begin{gathered}
\tau_{z} I_{\alpha}\left(. ; q^{2}\right)(w)=I_{\alpha}\left(z ; q^{2}\right) I_{\alpha}\left(w ; q^{2}\right) \\
M_{z} I_{\alpha}\left(. ; q^{2}\right)(w)=I_{\alpha}\left(w z ; q^{2}\right) I_{\alpha}\left(w ; q^{2}\right)
\end{gathered}
$$

Remark 5: If $q \rightarrow 1^{-}$, we obtain the generalized translation operator given in ([3], page 181).

Proposition 2: Let $f(z)=\sum_{n=0}^{\infty} a_{n} z^{2 n} \in \mathbb{F}_{q, \alpha}$ and $z, w \in D\left(o, \frac{1}{1-q}\right)$. Then

1)

$$
\begin{aligned}
\tau_{z} f(w)= & \sum_{n=0}^{\infty} a_{n}\left[\sum_{k=0}^{n}\left(\begin{array}{l}
n \\
k
\end{array}\right)_{q^{2}}\right. \\
& \left.\cdot \frac{\Gamma_{q^{2}}(\alpha+1) \Gamma_{q^{2}}(n+\alpha+1)}{\Gamma_{q^{2}}(k+\alpha+1) \Gamma_{q^{2}}(n-k+\alpha+1)}\left(\frac{z}{w}\right)^{2 k}\right] w^{2 n} .
\end{aligned}
$$

2) $M_{z} f(w)=\sum_{n=0}^{\infty}\left[\sum_{k=0}^{n} \frac{a_{n-k}}{b_{2 k}\left(\alpha ; q^{2}\right)} z^{2 k}\right] w^{2 n}$. 
Proof. 1) Let $f(z)=\sum_{n=0}^{\infty} a_{n} z^{2 n} \in \mathbb{F}_{q, \alpha}$. From (14), we have

$$
\tau_{z} f(w)=\sum_{n=0}^{\infty} \frac{\Delta_{q, \alpha}^{n} f(w)}{b_{2 n}\left(\alpha ; q^{2}\right)} z^{2 n} ; \quad w, z \in D\left(o, \frac{1}{1-q}\right)
$$

Since from Lemma 1, 2),

$$
\Delta_{q, \alpha}^{n} w^{2 k}=\frac{b_{2 k}\left(\alpha ; q^{2}\right)}{b_{2(k-n)}\left(\alpha ; q^{2}\right)} w^{2(k-n)}, \quad k \geq n
$$

we can write

$$
\Delta_{q, \alpha}^{n} f(w)=\sum_{k=n}^{\infty} a_{k} \frac{b_{2 k}\left(\alpha ; q^{2}\right)}{b_{2(k-n)}\left(\alpha ; q^{2}\right)} w^{2(k-n)}
$$

Thus we obtain

$$
\tau_{z} f(w)=\sum_{n=0}^{\infty} a_{n} \sum_{k=0}^{n} \frac{b_{2 n}\left(\alpha ; q^{2}\right)}{b_{2 k}\left(\alpha ; q^{2}\right) b_{2(n-k)}\left(\alpha ; q^{2}\right)} w^{2(n-k)} z^{2 k}
$$

On the other hand from (1) and (2), we get

$$
\begin{aligned}
& \frac{b_{2 n}\left(\alpha ; q^{2}\right)}{b_{2 k}\left(\alpha ; q^{2}\right) b_{2(n-k)}\left(\alpha ; q^{2}\right)} \\
& =\left(\begin{array}{l}
n \\
k
\end{array}\right)_{q^{2}} \frac{\Gamma_{q^{2}}(\alpha+1) \Gamma_{q^{2}}(n+\alpha+1)}{\Gamma_{q^{2}}(k+\alpha+1) \Gamma_{q^{2}}(n-k+\alpha+1)}
\end{aligned}
$$

which gives the 1).

2) From (15), we have

$$
M_{z} f(w)=\sum_{n=0}^{\infty} \frac{M^{n} f(w)}{b_{2 n}\left(\alpha ; q^{2}\right)} z^{2 n} ; \quad w, z \in D\left(o, \frac{1}{1-q}\right)
$$

But from (12), we have

$$
M^{n} f(w)=\sum_{k=n}^{\infty} a_{k-n} w^{2 k}
$$

Thus we obtain

$$
M_{z} f(w)=\sum_{n=0}^{\infty}\left[\sum_{k=0}^{n} \frac{a_{n-k}}{b_{2 k}\left(\alpha ; q^{2}\right)} z^{2 k}\right] w^{2 n}
$$

According to Theorem 2 we study the continuous property of the operators $T_{z}$ and $M_{z}$ on $\mathbb{F}_{q, \alpha}$.

Theorem 4: If $f \in \mathbb{F}_{q, \alpha}$ and $z \in D\left(o, \frac{1}{1-q}\right)$, then $T_{z} f$ and $M_{z} f$ belong to $\mathbb{F}_{q, \alpha}$, and we have

1) $\left\|T_{z} f\right\|_{\mathbb{F}_{q, \alpha}} \leq I_{\alpha}\left(\frac{|z|}{\sqrt{1-q}} ; q^{2}\right)\|f\|_{\mathbb{F}_{q, \alpha}}$,
2) $\left\|M_{z} f\right\|_{\mathbb{F}_{q, \alpha}} \leq I_{\alpha}\left(\frac{|z|}{\sqrt{1-q}} ; q^{2}\right)\|f\|_{\mathbb{F}_{q, \alpha}}$.

Proof. From (14) and Theorem 2, 1), we deduce

$$
\begin{aligned}
\left\|T_{z} f\right\|_{\mathbb{F}_{q, \alpha}} & \leq \sum_{n=0}^{\infty}\left\|\Delta_{q, \alpha}^{n} f\right\|_{\mathbb{F}_{q, \alpha}} \frac{|z|^{2 n}}{b_{2 n}\left(\alpha ; q^{2}\right)} \\
& \leq \sum_{n=0}^{\infty} \frac{|z|^{2 n}}{(1-q)^{n} b_{2 n}\left(\alpha ; q^{2}\right)}\|f\|_{\mathbb{F}_{q, \alpha}}
\end{aligned}
$$

Therefore,

$$
\left\|T_{z} f\right\|_{\mathbb{F}_{q, \alpha}} \leq I_{\alpha}\left(\frac{|z|}{\sqrt{1-q}} ; q^{2}\right)\|f\|_{\mathbb{F}_{q, \alpha}}
$$

which gives the first inequality, and as in the same way we prove the second inequality of this theorem.

From Proposition 1 we deduce the following results.

Proposition 3: For all $f, g \in \mathbb{F}_{q, \alpha}$, we have

$$
\begin{aligned}
& \left\langle M_{z} f, g\right\rangle_{\mathbb{F}_{q, \alpha}}=\left\langle f, T_{z} g\right\rangle_{\mathbb{F}_{q, \alpha}} \\
& \left\langle T_{z} f, g\right\rangle_{\mathbb{F}_{q, \alpha}}=\left\langle f, M_{-} g\right\rangle_{\mathbb{F}_{q, \alpha}}
\end{aligned}
$$

We denote by $R_{z}$ the following operator defined on $\mathbb{F}_{q, \alpha}$ by

$$
\begin{aligned}
R_{z}:=T_{\bar{z}} M_{z}-M_{\bar{z}} T_{z} & =I_{\alpha}\left(\bar{z} \Delta_{q, \alpha}^{1 / 2} ; q^{2}\right) I_{\alpha}\left(z M^{1 / 2} ; q^{2}\right) \\
& -I_{\alpha}\left(\bar{z} M^{1 / 2} ; q^{2}\right) I_{\alpha}\left(z \Delta_{q, \alpha}^{1 / 2} ; q^{2}\right)
\end{aligned}
$$

Then, we prove the following theorem.

Theorem 5. For all $f \in \mathbb{F}_{q, \alpha}$, we have

$$
\left\|M_{z} f\right\|_{\mathbb{F}_{q, \alpha}}^{2}=\left\|T_{z} f\right\|_{\mathbb{F}_{q, \alpha}}^{2}+\left\langle f, R_{z} f\right\rangle_{\mathbb{F}_{q, \alpha}}
$$

Proof. From Proposition 3, we get

$$
\begin{aligned}
\left\|M_{z} f\right\|_{\mathbb{F}_{q, \alpha}}^{2} & =\left\langle f, T_{z} M_{z} f\right\rangle_{\mathbb{F}_{q, \alpha}} \\
& =\left\langle f,\left(M_{\bar{z}} T_{z}+R_{z}\right) f\right\rangle_{\mathbb{F}_{q, \alpha}} \\
& =\left\|T_{z} f\right\|_{\mathbb{F}_{q, \alpha}}^{2}+\left\langle f, R_{z} f\right\rangle_{\mathbb{F}_{q, \alpha}}
\end{aligned}
$$

\section{References}

[1] V. Bargmann, “On a Hilbert Space of Analytic Functions and an Associated Integral Transform, Part I," Communications on Pure and Applied Mathematics, Vol. 14, No. 3, 1961, pp. 187-214. doi:10.1002/cpa.3160140303

[2] C. A. Berger and L. A. Coburn, "Toeplitz Operators on the Segal-Bargmann Space,” Transactions of the American Mathematical Society, Vol. 301, 1987, pp. 813-829. doi:10.1090/S0002-9947-1987-0882716-4 
[3] F. M. Cholewinski, "Generalized Fock Spaces and Associated Operators,” SIAM Journal on Mathematical Analysis, Vol. 15, No. 1, 1984, pp. 177-202. doi:10.1137/0515015

[4] A. Fitouhi, M. M. Hamza and F. Bouzeffour, "The $q-j_{\alpha}$ Bessel Function,” Journal of Approximation Theory, Vol. 115, No. 1, 2002, pp. 144-166. doi:10.1006/jath.2001.3645

[5] G. H. Jackson, “On a $q$-Definite Integrals,” The Quarterly Journal of Pure and Applied Mathematics, Vol. 41, 1910, pp. 193-203.

[6] T. H. Koornwinder, "Special Functions and $q$-Commuting Variables,” Fields Institute communications, Vol. 14, 1997, pp. 131-166. 